\title{
Correction to: Reliability of Field-Based Fitness Tests in Adults: A Systematic Review
}

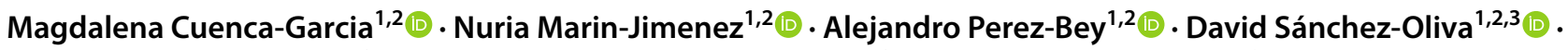 \\ Daniel Camiletti-Moiron ${ }^{1,2}$ (1) Inmaculada C. Alvarez-Gallardo ${ }^{1,2}$ (1) Francisco B. Ortega ${ }^{4,5,6}$ (D) \\ Jose Castro-Piñero ${ }^{1,2}$ (i)
}

Published online: 12 February 2022

(c) Springer Nature Switzerland AG 2022

\section{Correction to: Sports Medicine https://doi.org/10.1007/s40279-021-01635-2}

In this article the wrong figure appeared as Fig. 2. The Fig. 2 should have appeared as shown below.

The original article can be found online at https://doi.org/10.1007/ s40279-021-01635-2.

Nuria Marin-Jimenez

nuria.marin@uca.es

1 GALENO Research Group, Department of Physical Education, Faculty of Education Sciences, School of Education, University of Cádiz, Puerto Real, Avenida República Saharaui S/N, 11519 Puerto Real, Cádiz, Spain

2 Instituto de Investigación e Innovación Biomédica de Cádiz (INiBICA), Cadiz, Spain

3 ACAFYDE Research Group, Faculty of Sport Sciences, University of Extremadura, Caceres, Spain

4 PROFITH "PROmoting FITness and Health Through Physical Activity" Research Group, Sport and Health University Research Institute (iMUDS), Department of Physical and Sports Education, Faculty of Sport Sciences, University of Granada, 18071 Granada, Spain

5 Faculty of Sport and Health Sciences, University of Jyväskylä, Jyväskylä, Finland

6 Department of Biosciences and Nutrition, Karolinska Institutet, Huddinge, Sweden 


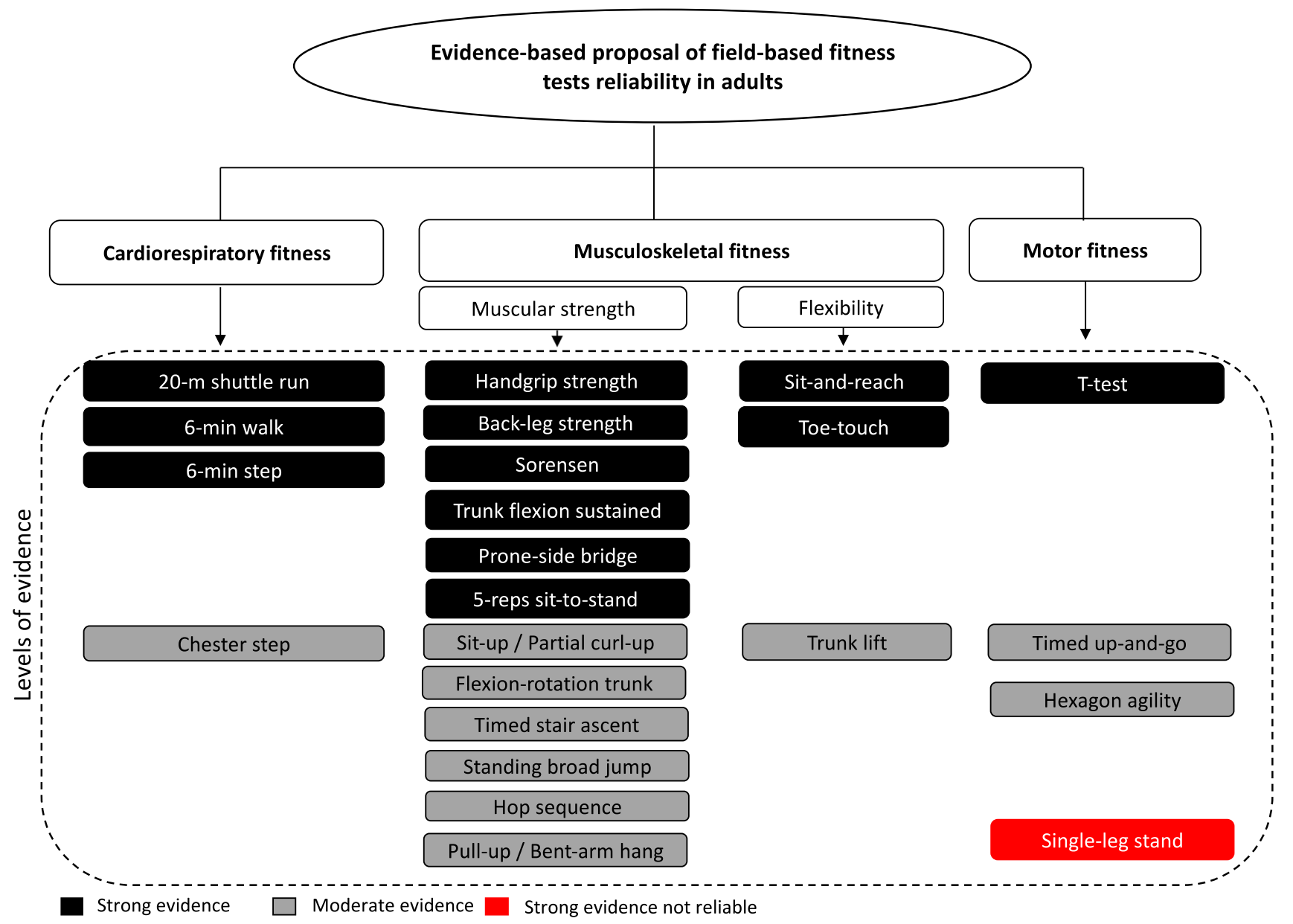

Fig. 2 Evidence-based proposal for field-based fitness tests reliability in adults

The original article has been corrected. 